\title{
Channeling in Bent Crystallites: A New Method to Enhance the Radiation Shielding Efficiency ${ }^{*}$
}

\author{
Mukhtar Ahmed Rana \\ Medical Equipment Research and Development Division (MERADD), ICCC, Lahore, Pakistan \\ Email: ranamssa@gmail.com
}

How to cite this paper: Rana, M.A. (2018) Channeling in Bent Crystallites: A New Method to Enhance the Radiation Shielding Efficiency. Modern Instrumentation, 7, $35-41$.

https://doi.org/10.4236/mi.2018.73004

Received: March 26, 2018

Accepted: July 16, 2018

Published: July 19, 2018

Copyright $\odot 2018$ by author and Scientific Research Publishing Inc. This work is licensed under the Creative Commons Attribution International License (CC BY 4.0).

http://creativecommons.org/licenses/by/4.0/

\begin{abstract}
A new method is proposed here aiming at designing a shielding wall with the efficiency significantly higher than that of traditional designs. This new design arises from the idea of using channeling in multilayered shielding wall structure, each layer composed of bent crystallites distributed in a way that each layer covers a small section of $2 \pi$ angular range of which wall is exposed. Part of the incident charged particles will get channeled in bent crystallites in each layer. Bending of channeled particles in bent crystallites will change their directions in the wall increasing their path lengths in the wall which would enhance its shielding efficiency for charged particle radiations. Proposed design is useful for radiation shielding in fission power plants, future fusion reactors and air travel.
\end{abstract}

\section{Keywords}

Radiation Shielding, Bent Crystals, Channeling, Nuclear and Thermonuclear Reactors, Cosmic Rays

\section{Introduction}

Radiation shielding is a great concern in environments of nuclear technology and air travel [1] [2]. These environments include various radiation fields with a considerable component of charged particle rays. Charged particle rays include fission fragments, heavy and light ions and nuclei, and electrons. A major fraction of these particles from nuclear/thermonuclear reactors and cosmic rays has high energy loss rate in tissue and other materials. Shielding of human beings and material devices against their radiation is highly desirable. There are several radiation shielding designs using numerous materials of special characteristics,

${ }^{*}$ A new method for radiation shielding. 
but none of them have made use of the concept presented here with the theme as "bending charged particle radiations in the shields to increase their path lengths in them". Bending of charged particle rays would be achieved by employing a multilayered shield with each layer composed of pieces of bent crystals. Strong fields of bent crystalline planes and axes have an ability of bending charged particles entering planar and axial channels under certain conditions [3] [4] [5] [6]. Details of the method and its implementation are given below. Section 2 describes channeling condition in bent crystals whereas proposed method for enhancement of shielding efficiency is given in Section 3. Section 4 includes conclusion of the study.

\section{Channeling in Bent Crystals}

Ion channeling is the passage of a particle beam (particles travelling in parallel) through open spaces in materials, e.g., crystal planar and axial channels or possibly nanotubes. Traditionally, this process is studied in single crystals where a variety of channels, both with similar and very different geometric and electronic structures, are available. The channeled beam in a crystal travels on a very specific path with a statistical variation. Ion channeling was discovered in early 1960s and it continued developing due to a number of its present [7] [8] and potential uses including channeling in bent crystals [3] [9] [10] and defect measurement in crystals [11] [12] [13] [14] [15].

Steering of channelled particles in a crystal continues even if the crystal is slightly bent deviating from their original direction as in strong magnetic fields of strength several thousand tesla. This fact provides us with the possibility of designing a "crystalline kicker" (Uggerhøj, 2005) [3]. This crystalline kicker for charged particles is shown in Figure 1.

Lindhard [16] has shown that incident angle $\left(\theta_{i}\right)$ of the particle trapped in transverse field of the crystalline channel must not exceed the limiting angle, called Lindhard angle $\left(\theta_{L}\right)$, as given below,

$$
\theta_{i} \leq \theta_{L}=\sqrt{\frac{2 U_{T}}{\varepsilon_{\|}}},
$$

where $U_{T}$ is the transverse potential of the channel and $\varepsilon_{\|}$is longitudinal kinetic energy of the particle in the channel and is given by,

$$
\varepsilon_{\|}=m_{o} c^{2} \gamma
$$

where $\gamma$ is the well known Lorentz factor. Under the condition given in Equation (1), transverse kinetic energy of the channeled particle will remain below the transverse potential $U_{T}$. Let $\theta_{S O}$ is the angle of the curvature in the bent crystal subtended on length of one transverse oscillation of the planar channeled particle $\left(L_{S O}\right)$ so that $L_{S O}=r \theta_{S O}$, where $r$ is radius of curvature of the bent crystal. Channeling in the bent crystal will persist if,

$$
\theta_{S O} \leq \theta_{L} .
$$




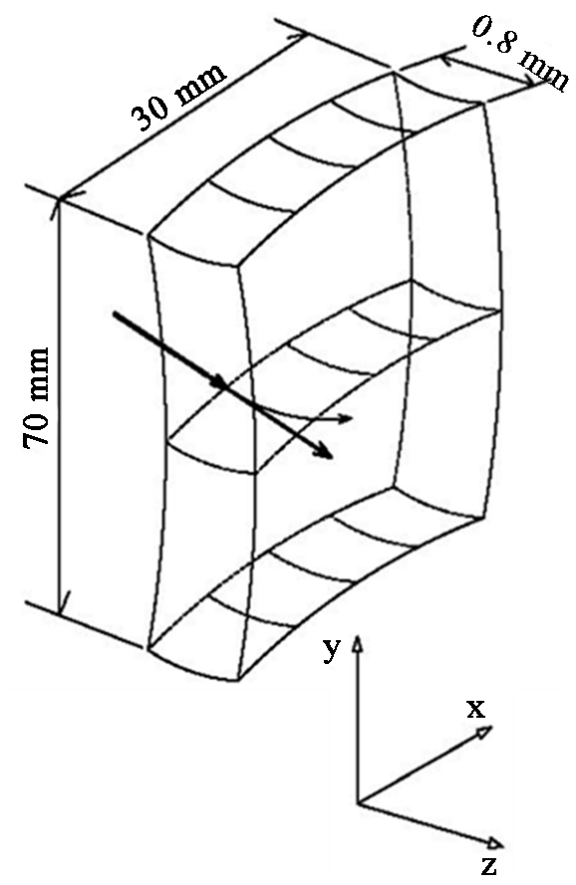

Figure 1. A schematic drawing showing bending of the channeled particles in the bent crystal. Typical dimensions are shown.

Maximum bending angle of the channeled particle $\left(\theta_{\max }\right)$ in the bent crystal is given by the following condition [17],

$$
\theta_{\max } \leq \theta_{L} N_{O s c}
$$

where $N_{O s c}$ is the number of transverse oscillations made by the channeled particle in the bent channel of the crystal. Above Equations (1)-(4) describe completely the condition of channeling in the bent crystal for the purpose being served in this paper.

\section{Proposed Method for Enhancement of Shielding Efficiency}

It is shown in Section 2 that charged particles entering channels of a bent crystal under certain conditions would bend by an angle up to $\theta_{\max }$ (Equation (4)) which depends on Lindhard angle $\theta_{L}$ and number of transverse oscillations made by the channeled particle $N_{O s c}$. Value of $\theta_{L}$ is determined by properties of the crystalline material and used channel along with characteristics of the channeled particle like atomic and mass numbers, charge state and energy. The number of oscillations $N_{O s c}$ can be controlled by length of the bent crystal. Let us now consider a test piece of a bent crystal (like Ge) with length $L_{T}$ and Lindhard critical angle $\theta_{T}^{L}$ for a certain radiation (say proton, alpha particle or a fission fragment). The new scientific idea being presented here is that a multilayered radiation shield with each layer composed of bent crystalline pieces arranged in a certain manner will serve as a kicker for charged particle rays changing direction of the radiations and hence increasing their path lengths in the shield giving rise to the enhanced shielding efficiency. The maximum bend- 
ing angle $\left(\theta_{T}^{\max }\right)$ of a charged particle in the above mentioned bent crystal piece is given by,

$$
\theta_{T}^{\max } \leq \theta_{T}^{L} \cdot \frac{L_{T}}{L_{T}^{S O}},
$$

where labels "T" and "SO" refer to test crystal piece and single transverse oscillation of the channeled particle, respectively.

Figure 2 shows the detail of the design pictorially. Left section shows a straight crystal whereas right section two layers of the proposed shield. This scheme can be employed for the cases when radiation field falling on the shield wall has at least a local directionality which means charged particles falling on a small surface area of the shield wall will incident in a an angular range as shown in Figure 3. In Figure 3, a 2D shielding box of a radiation source is shown. A wall of infinite dimensions will have $2 \pi$ directions in which radiation can reach it. For a wall of limited dimensions, angular range will be much narrower than $2 \pi$. Angular range of radiations reaching each wall from the source may be divided into several sections, as divided into four sections in Figure 3. Particles falling in each such a section will have a limited angular range. In such cases above mentioned design will widen incident angular range of radiations falling on the wall increasing path lengths of radiations in the wall, hence enhancing shielding efficiency of the wall. In this scheme, bent crystalline pieces will be arranged in each layer in each section (I to IV) covering a section of the entire particle incidence range. Bent channels in bent crystals will offer entry into them to a part of radiations bending them in the shield. If a number of such layers, covering all the incidence range in a section, are employed in a structure, major fraction of incident charged rays will bend in the shield increasing path lengths of radiations in the shield. Two layers covering two small angular segments in the incidence range are shown in right column of Figure 2. In real case with $2 \mathrm{D}$

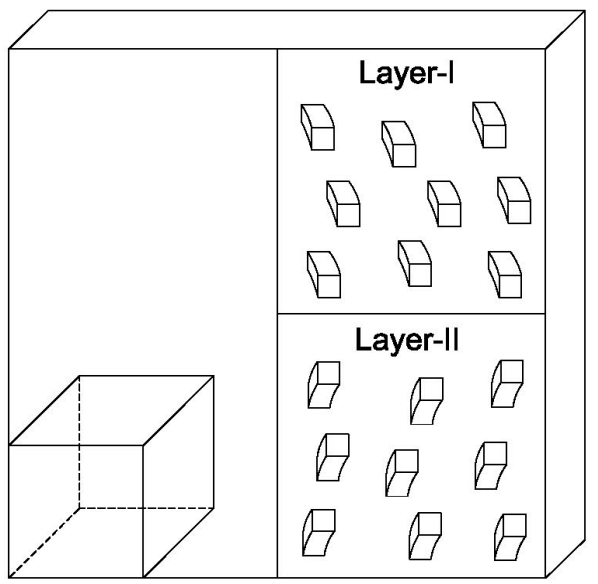

Figure 2. Design of the shielding wall of the charged particle radiations. Left part shows straight crystal structure whereas right part two layers of a multilayered shielding design with each layer composed of bent crystal pieces to bend a part of falling radiations. 


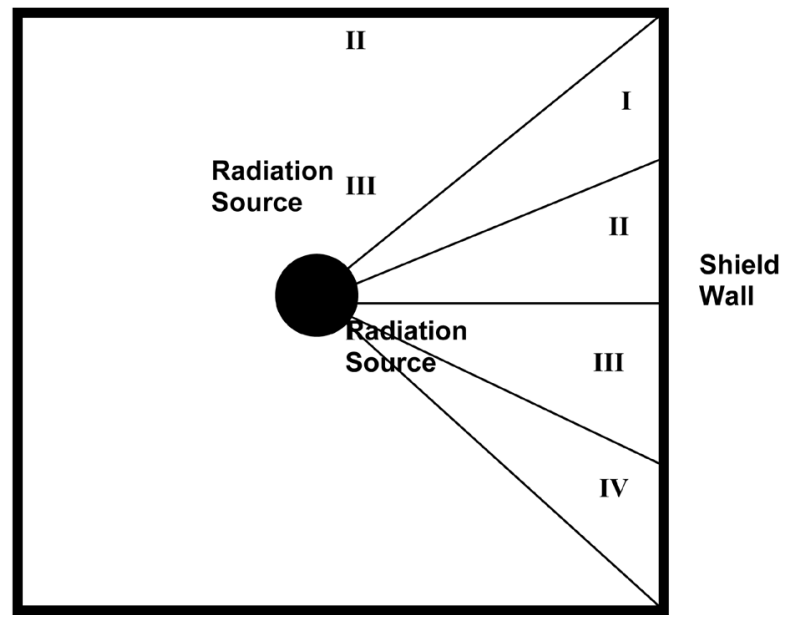

Figure 3. A charged particle radiation source enclosed by a shielding box (2D case for simplicity). Radiations falling on right wall are classified into four angular sections, each of them with a limited range of incidence angle of radiations falling on it.

shielding wall, wall surface will be divided in 2D grid with each grid element having a multilayered bent crystal structures. Alignment of crystal pieces will be arranged according to the range of incidence angles of charged particle rays. These bent crystal pieces will have length from fraction of a millimeter to tens of a millimeter depending upon charge and energy of radiations. Cross-sectional dimension of these elements will have inverse relationship with bending efficiency. This shield design will show efficiency better than the traditional shield wall. High efficiency amorphous material layers can also be joined behind this new multilayered structure for better shielding.

\section{Conclusion}

A new charged particle radiation shield design is proposed here. This design will have a higher shielding efficiency than the tradition designs. In the new design, multilayered wall structure with each wall composed of bent crystal pieces is proposed. Each layer will bent a part of charged particle rays entering in bent crystals under channeling condition increasing path lengths of radiations in the shield. If a sufficient number of layers are used, major fraction of charged particles will be bent in the shield travelling longer distance in the shield compared with the traditional design, hence enhancing shielding efficiency. This shielding design can be employed in a number of radiation environments with situations comparable to that described in Figure 3. It may be noticed that the present design is different but has some similarity with a proposal by Breese [18]. This design will consume shielding materials in a smaller quantity compared to the traditional design.

\section{Acknowledgements}

I express my gratitude to my Ph.D. supervisors/guides Prof. Breese, M.B.H., As- 
soc. Prof. Osipowicz, T., Prof. Watt, F. and Dr. Ren, M.Q. at the National University of Singapore, for guidance and discussions. This idea was initially discussed with Dr. Valery Biryukov (Russia) in 2008. Email discussions with several colleagues worldwide are gratefully acknowledged for understanding basics of ion channeling. Leading colleagues in this regard include Dr. Scandale, W., Dr. Biryukov, V.M., Dr. Taratin, A.M., Moller, S.P., Dr. Mazzolari, A., Greiner, W. and Fromm, M.

\section{References}

[1] Ewing, R.C. (2007) Materials Science: Displaced by Radiation. Nature, 445, 161-162. https://doi.org/10.1038/445161a

[2] Farnan, I., Cho, H. and Weber, W.J. (2007) Quantification of Actinide $\alpha$-Radiation Damage in Minerals and Ceramics. Nature, 445, 190-193.

https://doi.org/10.1038/nature05425

[3] Uggerhøj, U.I. (2005) The Interaction of Relativistic Particles with Strong Crystalline Fields. Review of Modern Physics, 77, 1131-1172.

https://doi.org/10.1103/RevModPhys.77.1131

[4] Møller, S.P. (1995) High-Energy Channeling-Applications in Beam Bending and Extraction. Nuclear Instruments and Methods in Physics Research Section A, 361, 403-420. https://doi.org/10.1016/0168-9002(95)00181-6

[5] Baurichter, A., et al. (2000) Channeling of High-Energy Particles in Bent Crystals-Experiments at the CERN SPS. Nuclear Instruments and Methods in Physics Research Section B, 164-165, 27-43. https://doi.org/10.1016/S0168-583X(99)01062-9

[6] Biryukov, V.M., et al. (1994) On Measuring $70 \mathrm{GeV}$ Proton Dechanneling Lengths in Silicon Crystals (110) and (111). Nuclear Instruments and Methods in Physics Research Section B, 86, 245-250. https://doi.org/10.1016/0168-583X(94)95286-8

[7] Feldman, L.C., Mayer, J.W. and Picraux, S.T. (1982) Materials Analysis by Ion Channeling: Submicron Crystallography. Academic Press, New York.

[8] Breese, M.B.H., Jemieson, D.N. and King, P.J.C. (1996) Materials Analysis Using a Nuclear Microprobe. John Wiley \& Sons, New York.

[9] Scandale, W., et al. (2008) Apparatus to Study Crystal Channeling and Volume Reflection Phenomena at the SPS H8 Beamline. Review of Scientific Instruments, 79, Article ID: 023303. https://doi.org/10.1063/1.2832638

[10] Rana, M.A. (2008) A New Method for Monitoring the Radiation Damage in Nuclear Waste Containers Using Ion Channeling. Annals of Nuclear Energy, 35, 1580-1583. https://doi.org/10.1016/j.anucene.2008.01.015

[11] Breese, M.B.H., Teo, E.J., Rana, M.A., Huang, L., van Kan, J.A., Watt, F. and King, P.J.C. (2004) Observation of Many Coherent Oscillations for MeV Protons Transmitted through Stacking Faults. Physical Review Letters, 92, Article ID: 045503. https://doi.org/10.1103/PhysRevLett.92.045503

[12] Rana, M.A., Breese, M.B.H. and Osipowicz, T. (2004) A Monte Carlo Simulation Study of Channelling and Dechannelling Enhancement Due to Lattice Translations. Nuclear Instruments and Methods in Physics Research Section B, 222, 53-60. https://doi.org/10.1016/j.nimb.2003.12.081

[13] Lu, Y., Cong, G.W., Liu, X.L., Lu, D.C. and Wang, Z.G. (2004) Depth Distribution of the Strain in the GaN Layer with Low-Temperature AlN Interlayer on Si(111) 
Substrate Studied by Rutherford Backscattering/Channeling. Applied Physics Letters, 85, 5562. https://doi.org/10.1063/1.1830679

[14] Rana, M.A. (2008) Planar Channelling Criticalities of MeV Protons in Si Crystal: Simulations, Evaluation and Applications. Chinese Physics Letters, 25, 3724-3727. https://doi.org/10.1088/0256-307X/25/10/056

[15] Wang, K., Ding, Z. and Yao, S. (2007) Elastic Strain in $\mathrm{Mg}_{0.28} \mathrm{Zn}_{0.72} \mathrm{O}$ Layer: Combined Rutherford Backscattering/Channeling and X-Ray Diffraction. Nuclear Instruments and Methods in Physics Research Section B, 259, 966-968. https://doi.org/10.1016/j.nimb.2007.03.008

[16] Lindhard, J. (1965) Influence of Crystal Lattice on Motion of Energetic Charged Particles. Kongel. Dan. Vidensk. Selsk., Mat.-Fys. Medd, 34, 1.

https://www.osti.gov/biblio/4536390

[17] Solov'yov, A.V., Schäfer, A. and Greiner, W. (1996) Channeling Process in a Bent Crystal. Physical Review E, 53, 1129-1137. https://doi.org/10.1103/PhysRevE.53.1129

[18] Breese, M.B.H. (2007) A Large-Area Bent Crystal Shield for Deflection of High-Energy Ions. Applied Physics Letters, 91, Article ID: 261901.

https://doi.org/10.1063/1.2824457 